\title{
Dynamic Approach and Testbed for Small and Medium Players Simulation in Smart Grid Environments
}

\author{
Luis Gomes*. Haroldo L. M. Amaral**.Filipe Fernandes* \\ Pedro Faria*. Zita Vale*. Carlos Ramos*. André N. de Souza** \\ * GECAD - Knowledge Engineering and Decision Support Research Center \\ IPP - Polytechnic Institute of Porto, Porto, Portugal; (e-mail: \{lufog, fijgf, pnfar, zav,csr\}@isep.ipp.pt). \\ ** LSISPOTI - Laboratory of Power Systems and Intelligent Techniques \\ UNESP - Universidade Estadual Paulista, Bauru, São Paulo, Brazil; (e-mail: agaelema@gmail.com, andrejau@feb.unesp.br).
}

\begin{abstract}
The Smart Grid environment allows the integration of resources of small and medium players through the use of Demand Response programs. Despite the clear advantages for the grid, the integration of consumers must be carefully done. This paper proposes a system which simulates small and medium players. The system is essential to produce tests and studies about the active participation of small and medium players in the Smart Grid environment. When comparing to similar systems, the advantages comprise the capability to deal with three types of loads - virtual, contextual and real. It can have several loads optimization modules and it can run in real time. The use of modules and the dynamic configuration of the player results in a system which can represent different players in an easy and independent way. This paper describes the system and all its capabilities.
\end{abstract}

\section{INTRODUCTION}

The International Energy Agency recently concluded that smart grids could "coordinate the needs and capabilities of all generators, grid operators, end-users, and electricity market stakeholders to operate all parts of the system as efficiently as possible, minimizing costs and environmental impacts while maximizing system reliability, resilience and stability" (International Energy Agency, 2011). For these reasons, the concept of smart grids has gained attention of politicians around the world (Brandstatt et al., 2012).

One of the advantages of smart grids is the active participation of small and medium players. The consumers will become a part of the smart grid management, having bilateral connections with other players of the grid (Ye Yan et al., 2013). To achieve a feasible and fast integration of small and medium consumers, Demand Response (DR) programs that can be launch by several players inside the smart grid are used (Yunfei Wang, 2011).

The integration of DR to allow an active participation of small and medium players must be studied. The consumer's action determines the success of the DR program. To provide satisfactory answers, consumers can implement a Demand Side Management system (Yee Wei Law et al., 2012). Studies regarding DSM in facilities can be seen in (Barbato, 2012), (Quintero, 2012) and (Guo et al., 2008).

DR programs represents mainly the reduction of consumption by small and medium players, which leads to load optimization algorithms. In (Barbato, 2012) an optimization process capable of minimizing the overall consumption is shown. The simulator proposed in (Guo et al., 2008) has the ability of comparing more than one optimization process. This ability is an advantage for the study of DSM.
This paper will propose a dynamic system capable of simulating small and medium players in smart grid environments. The simulator proposed has the goal of representing the actions and reactions of small and medium players. To perform a realistic representation of real consumers, the simulator has got three different types of loads: two of them are simulations, and the third comprises real loads connected to the system. The capacity of integrating real loads separates this simulator from the simulators proposed by other authors. The communications between real loads and the simulator are made through the TCP/IP protocol, to communicate with PLC loads, and/or through ZigBee protocol, for real loads that are connected to a management and controller devices.

To allow the DSM in the system, this simulator can accommodate multiple optimization algorithms, such as the simulator proposed in (Guo et al., 2008). However, the simulator proposed in this paper can easily integrate any optimization algorithm implemented in MATLAB.

The simulator proposed in this paper has the particularity of dealing with real loads. For this reason, the simulator may gain the functionality of a unique real system for the DSM. To allow the control and management of this system, a mobile application was developed. The focus in this paper is the control of a refrigerator that contains a ZigBee device capable of communicating with the simulator.

After the introduction, this paper presents the system architecture in section 2, followed by the description of the type of loads in section 3. Section 4 presents the implemented mobile application and a practical implementation. A case study regarding the analysis of the consumptions of a real refrigerator in our lab is shown in section 5. Finally, section 6 presents the conclusions of the paper. 


\section{SYSTEM ARCHITECTURE}

The proposed system simulates small and medium players that can be introduced in a smart grid environment. Smart grids are an important concept for the future of energy grids (Brandstatt et al., 2012). To achieve the complete implementation of smart grids it is necessary to test and analyse the integration of small and medium players, which represent most of the stakeholders in the grid.

The simulator described in this paper aims the creation of a testbed system which can realistically simulate small and medium players. For this purpose, the simulator has the possibility of integrating real loads. One can see the architecture of the simulator in Fig. 1.

The simulator is implemented in Java, yet it also uses MATLAB and GAMS. The use of MATLAB and GAMS was one requirement of the system, as these languages are essential for the execution of the system's optimization algorithms. The integration between MATLAB and Java was made by using MATLAB Builder JA, which allows the transformation of MATLAB functions into Java functions.

The optimization module is an essential part of the simulator. The simple management and control of loads are not enough to bring small and medium players into the smart grid environment. These players need to acquire some kind of intelligence, which has been introduced into the simulator by applying artificial intelligence techniques. Although being an important part of the simulator, the optimizations and the application of Artificial Neural Networks (ANN) in the simulator are not discussed in this paper. The focus is given to present the architecture of the simulator and the integration of real loads, namely the integration (in real time) of a real refrigerator into the simulator.

The optimizations modules and the optimization algorithms can be seen in (Gomes et al., 2013a); the use of ANN to achieve the user preferences according to the context can be seen in (Gomes et al., 2011); the combination of ANN and optimization allow a custom cut in the consumption, minimizing the negative impact for the users.

The intelligence of the system, provided mainly by the optimization algorithms, acts on the loads controlled by the simulator. The simulator supports three types of loads: Simulated Load; Contextual Load; and Real Load. The real loads are divided into two types: Programmable Logic Controller (PLC) Loads, and ZigBee Loads. The description of loads can be found in section 3 .

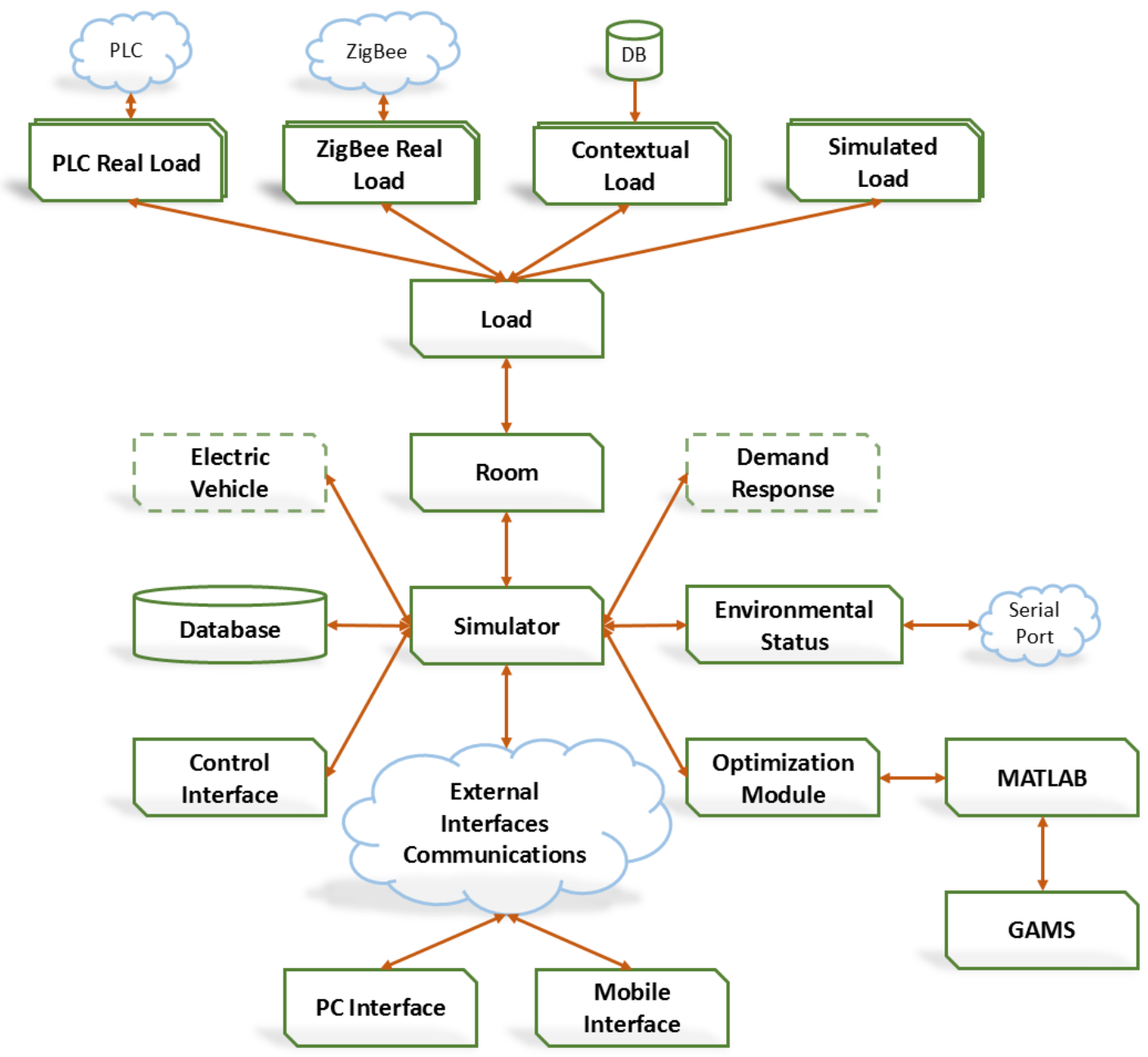

Fig. 1. Architecture of the simulator 
In the centre of the architecture it is possible to see the core of the simulator. All the modules were developed to work with the core to achieve the main goal of producing a realistic simulation of small and medium players.

The system modules with dashed lines are not yet entirely integrated in the simulator. They were implemented as prototypes in order to be lately integrated in the simulator.

To record previous consumptions and prices of energy executed by the grid operator, the system uses a SQL Server database. To allow the simulation of a facility, the simulator has a module for the environmental status of the facility. This module will receive real data from a device connected through a serial port. At the moment, it is possible to change the environmental status during the execution by using an interface, in which we can change, for example, the brightness, the temperature and the position of the people inside the facility.

The simulator does not have an interface capable of controlling the loads and the optimizations. It was developed an external interface communication module, in which interfaces that had been developed separately from the simulator can be connected.

The optimization module comprises the intelligence present in the system (Gomes et al., 2011). During a demand response program launched in the Smart Grid, the request for a cut in consumption inside the facilities is usual. This reduction of consumption can occur at any time, so it does not demand the permanent presence of an administrator in the facility. The autonomy in the facility is almost mandatory.

The simulator was developed to enable the easy integration of optimization algorithms implemented in MATLAB and GAMS. The use of this methodology allows the simulator to compare optimization algorithms and analyse their impacts in the small and medium player simulated. As this simulator can be easily integrated in a multi-agent system (Gomes et al., $2013 b$ ), it is also possible to analyse the impacts on the grid.

\section{TYPES OF LOADS SIMULATION}

The simulator described in section 2 deals with three types of loads (virtual, contextual and real), and with the decomposition of real loads of two types (PLC and ZigBee). Moreover, the system separates discrete loads from variable loads, making a total of eight variations of loads. Each of these variations represents a class in Java and it processes their own specifications. The control, monitoring, communication and simulation of loads is done by several threads, one for each load, making possible to simulate and control real loads during a real-time simulation run. Each thread represents a load and it is responsible for the interface between the simulator and the loads. Without this multithread approach, it is almost impossible to implement a realtime simulator capable of having real and simulated loads motorized and controlled in real time.

\subsection{Discrete Simulated Load}

This load is purely simulated by the system, and it can only be turned on or off. The load does not allow the flux control of the energy consumption. The simulation is done using fixed values of consumption. If the load is turned off, the consumption is zero; in case of the load is turned on, the consumption will be the maximum that the load can handle. The change between zero and the maximum consumption is instantaneous. This simulation can be used for loads, such as lamps and alarm clocks.

\subsection{Discrete Contextual Load}

Despite the discrete contextual load is simulated like the discrete simulated load, it has a different simulation procedure. This load will follow real measurements that were previously recorded into a file. To perform the simulation, the measures recorded must be from a real load; the measures must contain the full cycle of the load work (if we are dealing with loads, such as washing machines) or an extensive amount of measures, so they do not repeat constantly the same consumption values.

By using real measures, the system can simulate the loads that for some reason are not available in the laboratory. In this type of load, it is only possible to have loads that do not allow the variation of the flux of the energy consumption. Loads such as refrigerators or microwaves can be integrated into the simulation using a discrete contextual load.

\subsection{Discrete ZigBee Load}

ZigBee is a protocol of communications created by the ZigBee Alliance. The goal of this protocol was to define a standard of communications. The advantages of using this protocol comprise the security, standardization, and the low consumption of the equipment. The simulator described in this paper has an external xBee module that allows the communication with other equipment that have the same communication protocol.

Currently, there are two electronic devices to monitor and control loads wirelessly. The communications are made through the ZigBee protocol. These devices are capable of monitoring and controlling discrete loads which will be connected to the simulator. The discrete ZigBee loads will allow the system to use real loads in the simulation. Loads, such as televisions and water machines, can be controlled by the simulator using the class discrete ZigBee load.

\subsection{Discrete PLC Load}

In SCADA systems the presence of PLC equipment is usual. This equipment can have an Ethernet connection which can be used by an external system. The other system only needs to know the IP address of the PLC, and understand the protocol used by the specific PLC equipment. Although the use of PLC equipment is not widespread in residential housings, the use of PLC equipment in industrial facilities and laboratories is common.

The simulator described in this paper has the possibility of using loads that are connected to a PLC. The protocol used in the simulator is the ModBus, nevertheless others can easily be added. The class representative of the discrete PLC load can handle discrete loads, such as heat accumulators and fans. 


\subsection{Variable Simulated Load}

The class 'variable simulated load' is used to simulate loads that can adjust the energy consumption flux, for example, a regulated lamp. These types of loads are a challenge for the simulation because of the dynamic that brings to the system.

The loads in this class are entirely simulated by the system. The variation allowed is between $0 \%$ to $100 \%$, in which $0 \%$ represents the load without consumption, and $100 \%$ represents the maximum consumption allowed by the load. This class can simulate loads, such as engines and water pumps.

\subsection{Variable Contextual Load}

Some loads are impossible to be accommodated in the laboratory where the simulator is running. This can occur because of the size of the load, the danger that it may bring, the cost of acquisition and other aspects. In this cases, the simulator allows using consumption measures of real loads, leading to a more realistically simulation.

The file with the measures must have the consumption according to a period of time. The simulation will use this periods of time to simulate and change the load consumption. The periods can have any value defined by the users; however, the period must be according to the loads' variations. This type of load can simulate loads, such as industrial machinery and industrial furnaces.

\subsection{Variable ZigBee Load}

The variable ZigBee load allows the integration of real variable loads into the simulator. One of the devices developed can monitor and control variables loads. The principal is the same as the discrete ZigBee load communications use the ZigBee protocol.

The device can be connected to switches or sockets. After the connection, the device has the full control of the energy flow, and it can collect the consumption in real time. These devices can be connected to loads, such as air conditioners and boilers.

\subsection{Variable PLC Load}

Finally, the last variation of loads that the system can handle is the variable PLC load. This class was created to accommodate variable loads connected to PLC equipment.

The PLC works with memory positions and it has two types of data recorded in the memory (coil and register). To connect a load into a PLC, the user must define the memory positions associated with the load. Considering variable loads, the user must have a memory position for the load status (coil), a memory position for the consumption (register), and a memory position for the variation of the load (register). These memory positions must be configured in the simulator so it can successfully read the load data.

The loads that can be accommodated by this class are real loads, such as sound equipment and electric heaters.

\section{MOBILE APLICATION AND PRACTICAL IMPLEMENTATION}

The simulator deals with three types of loads with several variations, including real loads in the laboratory and in the building that can be integrated into the system. The integration of this features allowed the creation of a mobile application that can control not only the simulation parameters, but also real loads connected to the system.

The mobile application was developed for Android operating system and it connects to the simulator through the external interfaces communications module. The application was optimize for tablets with 10.1 inches and with a 1280x 800 resolution. In this application, the user can see the environmental status of the facility and the general information, such as the total consumption and the execution of demand response programs. The application allows controlling the loads configured in the simulator in real time.

It is also possible to execute the optimization algorithms allowed in the simulator. The entire interface of the application was dynamically built, allowing the adaptation of the application according to the simulator's configurations.

The system described was developed to control real loads and simulate loads, allowing the creation of a simulator for small and medium players. However, to analyse the real impacts caused by the smart grid integration, the system needed to transform itself into an intelligent demand side management system allowing not only the simulation, but also an independent system capable of monitoring and controlling real loads.

The real loads management, the optimization module and the mobile application allow the use of the system in real facilities. The applicability into real scenarios is tested and used regularly in our laboratory, where we can control wirelessly several loads connected to the system. This transforms the system into a hybrid system, in which we can produce a simulation or monitor and control real loads of a facility.

\section{CASE STUDY}

One of the possibilities of the system proposed in this paper is the use for lab tests and analysis. To prove the analytical capability of the system, this case study will analyse the consumptions of a refrigerator. It will be used a Discrete ZigBee Load to enable the integration of a normal refrigerator without the need for structural modifications. The device, working with ZigBee protocol, used in the refrigerator is shown in Fig. 4, in which the main modules of the device are presented.

The system allows any device that uses the ZigBee protocol. The original device presented in Fig. 2 only had a temperature sensor that needs to be placed inside the refrigerator. However, for this case study another temperature sensor placed outside of the refrigerator is used. This new temperature sensor is connected to the Signal Conditioning Board of the device. The data was recorded during an entire month. 
Using the data acquired from the measures, it is possible to retrieve the consumption profile of the refrigerator studied. This enables a better comprehension of the consumptions of the equipment, allowing a better management in future optimizations.

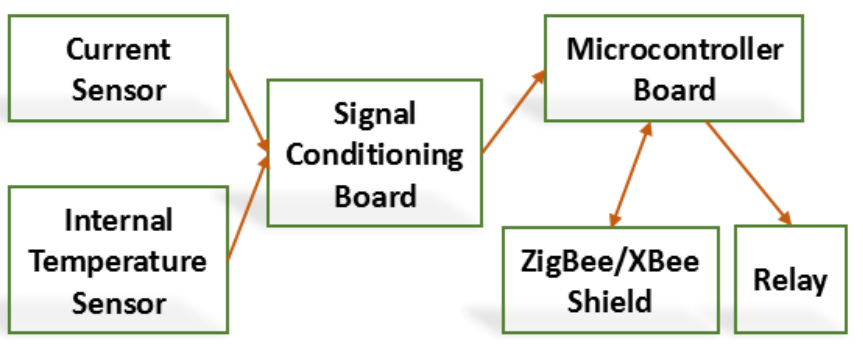

Fig. 2. Discrete ZigBee Load used in the case study

Fig. $4-a$ ) shows the profile of the refrigerator consumptions, internal temperatures, and external temperatures in the period between 00:30 p.m. and 10:40 p.m.. In order to evaluate the data received on the server, a filtering of the data was done to eliminate reading errors. On this profile it is clear that the lunch time occurs between 00:40 p.m. and 02:30 p.m., and the snack time occurs at 4:40 p.m.

The peaks occurred by opening the door, as this action turns on the internal lamp that has a consumption of $20 \mathrm{~W}$, and also increases the internal temperature that is shown in Fig. 4.

As one can see in Fig. $4-a$ ), in some periods the refrigerator turns the engine on to reduce the internal temperature. Every time the engine is activated, there is a peak signalling the initiation of the engine. This reaction is directly connected to the internal temperature of the refrigerator. The study of the profiles allows us to visualize the activation of the engine with the information on the internal temperature. This analysis enables managing the activation of the engine to be used on behalf of the simulator.

a)

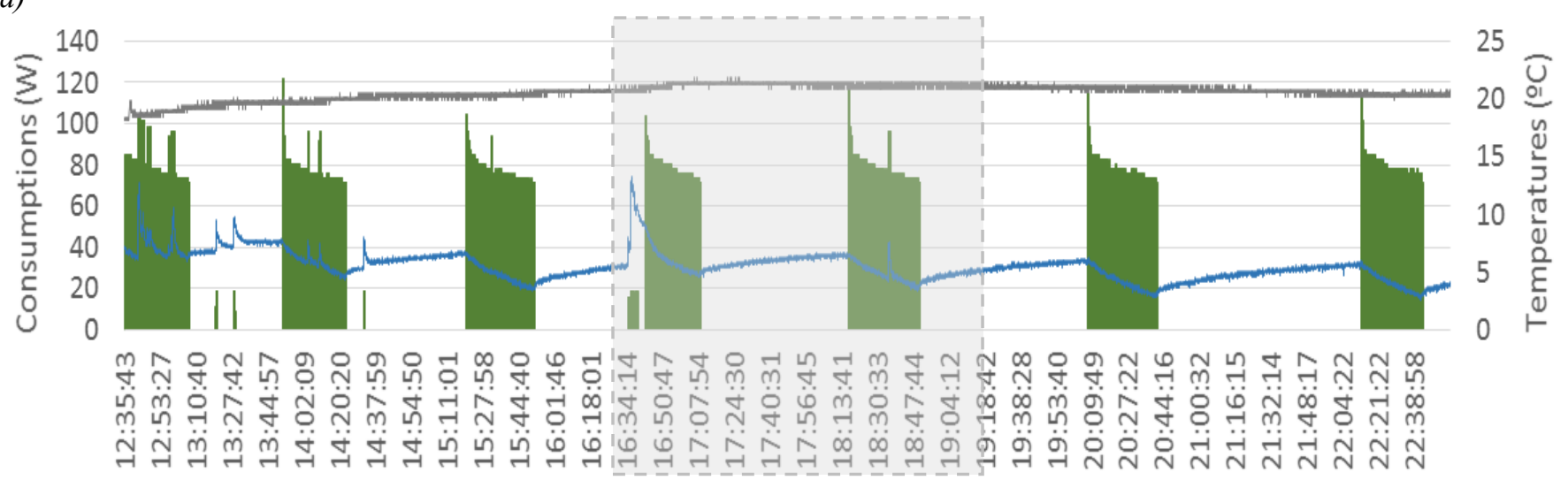

Hours

b)

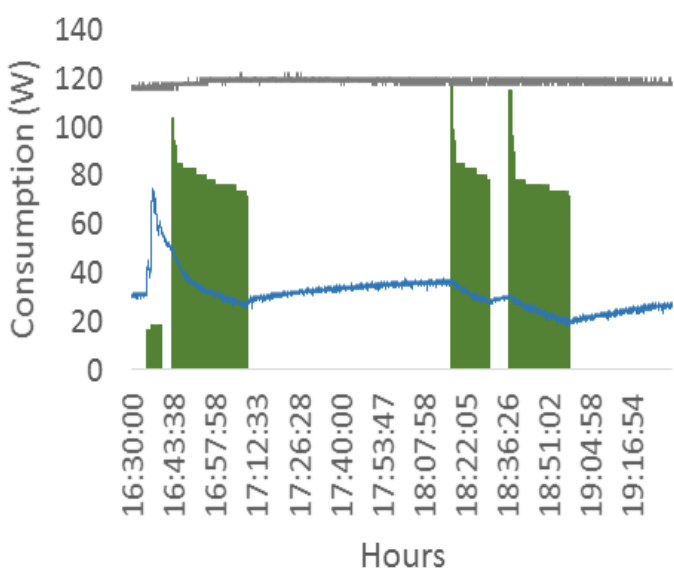

c)

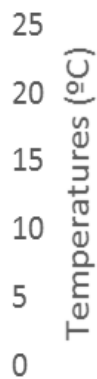

0

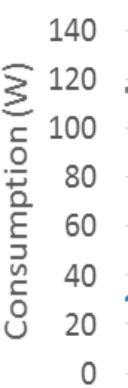

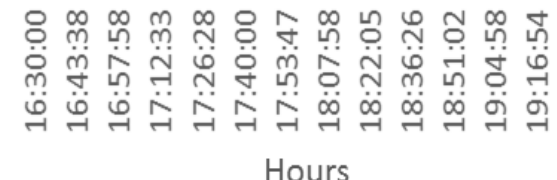

25

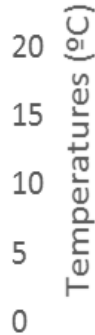

0

\section{Consumption Internal Temperature}

\section{- External Temperature}

Fig. 4. a) Refrigerator consumptions with the internal and external temperatures, b) unscheduled DR, c) scheduled DR

Knowing the profiles, the system can schedule the activation of the engine to match the needs of the people using the facility. The knowledge about the temperature behaviour inside the refrigerator allows the system to better manage the fulfilment of the refrigerator energy needs.
With the data collected, the system can analyse the users' profile, making adjustments to adapt the start of the engine to the users' needs. In this case study it has been considered the execution of a DR program at 6:30 p.m. with 5-minute duration. The user must participate in the program and, in 
order to optimize the resources, the refrigerator must be turned off. In Fig. $4-b$ ) is show the results of the implementation of the DR program without previous scheduling (DR event is has no advance notification). In this case, the engine will interrupt the refrigeration for 5 minutes.

Using the simulator presented to monitor the refrigerator, it is possible to learn the profile and adjust for scheduled DR programs. Without the user's profile, even scheduled DR programs result in cases such as the one presented in Fig. 4 $b$ ), in which it is necessary to cut loads in the middle of the load cycle.

Using the simulator and the information that it retrieves, it is possible to adjust scheduled DR programs. In Fig. $4-c$ ) the DR program launched at 6:30 p.m. was previously announced. Then the simulator extended the engine refrigeration time (at 4:45 p.m.), providing a lower temperature inside the refrigerator. The simulator even postponed for $6: 35 \mathrm{p} . \mathrm{m}$. the activation of the engine that usually starts at $6: 15$ p.m.. In this way, the refrigerator could stay off at 6:30 p.m. for the DR program participation.

\section{CONCLUSION}

The active participation of small and medium players in smart grid environments is essential to increase the reliability and efficiency in the grid. This active participation can be achieved by the application of demand response programs, and the implementation of demand side management in small and medium players.

To reach the reliability and efficiency expected, it is necessary to study the reactions and the impact in small and medium players. The participation is only accepted by the end consumer if it does not cause a negative impact in the facility. The proposed system allows the test and simulation of multiple scenarios considering small and medium players.

The system proposed allows dealing with several types of loads, resulting in a realistic testbed system and enabling the test of theoretical concepts in a complex simulator capable of using the most appropriate loads. The system proposed in this paper is able to compare concepts and methodologies, and it also enables becoming a real demand side management system that works with real loads of the facility.

\section{ACKNOWLEDGMENT}

This work is supported by FEDER Funds through COMPETE program and by National Funds through FCT under the projects FCOMP-01-0124-FEDER: PEstOE/EEI/UI0760/2011, and PTDC/SEN-ENR/122174/2010.

The research leading to these results has received funding from the People Programme (Marie Curie Actions) of the European Union's Seventh Framework Programme FP7/2007-2013/ under project ELECON - Electricity Consumption Analysis to Promote Energy Efficiency Considering Demand Response and Non-technical Losses, REA grant agreement No 318912.

\section{REFERENCES}

Barbato, A.; Carpentieri, G. (2012). Model and algorithms for the real time management of residential electricity demand. Energy Conference and Exhibition (ENERGYCON), 2012 IEEE International, pp.701,706.

Brandstatt, C.; Friedrichsen, N.; Meyer, R.; Palovic, M. (2012). Roles and responsibilities in smart grids: A country comparison. European Energy Market (EEM), 20129 th International Conference, pp.1,8.

Gomes, L.; Fernandes, F.; Sousa, T.; Silva, M.; Morais, H.; Vale, Z.; Ramos, C. (2011). Contextual intelligent load management with ANN adaptive learning module. International Conference on Intelligent System Applications to Power Systems - ISAP 2011.

Gomes, L.; Fernandes, F.; Faria, P.; Vale, Z.; Ramos, C.; Morais, H. (2013). Contextual Intelligent Load Management Considering Real-Time Pricing in a Smart Grid Environment. 17th International Conference on Intelligent System Applications to Power Systems - ISAP 2013.

Gomes, L.; Faria, P.; Morais, H.; Vale, Z.; Ramos, C. (2013). Distributed Agent-based Intelligent System for Demand Response Program Simulation in the Scope of Smart Grids. IEEE Intelligent Systems, Digital Object Identifier: 10.1109/MIS.2013.2.

Guo, Y.; Li, R.; Poulton, G.; Zeman, A. (2008). A Simulator for Self-Adaptive Energy Demand Management. SelfAdaptive and Self-Organizing Systems, 2008. SASO '08. Second IEEE International Conference, pp.64,73.

International Energy Agency, Technology Roadmap: Smart Grids, Par- is: IEA (2011).

Quintero M, C.G.; Mares, J.R.J. (2012). An analysis of Intelligent Demand Management criteria applied in a building case study. Alternative Energies and Energy Quality (SIFAE), 2012 IEEE International Symposium, pp.1,6.

U.S. Department of Energy Benefit of Demand Response in Electricity Market and Recommendations for Achieving Them (2006).

Ye Yan; Yi Qian; Sharif, H.; Tipper, D. (2013). A Survey on Smart Grid Communication Infrastructures: Motivations, Requirements and Challenges. Communications Surveys \& Tutorials, IEEE, vol.15 no.1, pp.5,20.

Yee Wei Law; Alpcan, T.; Lee, V.C.S.; Lo, A.; Marusic, S.; Palaniswami, M. (2012). Demand Response Architectures and Load Management Algorithms for Energy-Efficient Power Grids: A Survey. Knowledge, Information and Creativity Support Systems (KICSS), 2012 Seventh International Conference, pp.134,141.

Yunfei Wang; Pordanjani, I.R.; Wilsun Xu (2011). An EventDriven Demand Response Scheme for Power System Security Enhancement. Smart Grid, IEEE Transactions, vol.2 no.1, pp.23,29 